\title{
Oxygen Indicator Composed of an Organic/Inorganic Hybrid Compound of Methylene Blue, Reductant, Surfactant and Saponite
}

\author{
Makoto SumitanI, $*, * * *$ Shinsuke TaKagI, $* * *$ Yoshihiko Tanamura, $* * *$ and Haruo InOUE $*, * * \dagger$ \\ *Department of Applied Chemistry, Graduate Course of Engineering, Tokyo Metropolitan University, \\ 1-1 Minami-ohsawa, Hachiohji, Tokyo 192-0397, Japan \\ **CREST, Japan Science and Technology Agency, 4-1-8, Honcho, Kawaguchi, Saitama 332-0012, Japan \\ ***Tokyo Research Laboratory, Mitsubishi Gas Chemical Company, Inc., 1-1 Niijuku 6-chome, \\ Katsushika, Tokyo 125-0051, Japan
}

\begin{abstract}
An organic/inorganic hybrid compound consisting of methylene blue, a cationic surfactant and a reductant intercalated into saponite was found to serve as an oxygen indicator that changes color in the presence of oxygen. A mixture of a blue colored dye, methylene blue, a reductant in the form of ascorbic acid or reducing sugar, and cetyltrimethylammonium ion intercalated into synthetic saponite became colorless in an atmosphere having an oxygen concentration of less than 0.1 vol\%, and then returned to its blue color as a result of subsequent exposure to air. An oxygen indicator, in the form of a thin film coated on paper prepared by adding a pigment, phloxine B, to the above organic/inorganic hybrid compound, exhibited a pink color at oxygen concentrations of less than $0.1 \mathrm{vol} \%$, and a blue color at oxygen concentrations of higher than 0.5 vol\%. In addition, this oxygen indicator exhibited superior photo-fading resistance and storage stability compared with indicators using only methylene blue as the functional dye.
\end{abstract}

(Received March 8, 2004; Accepted May 31, 2004)

\section{Introduction}

The oxygen molecule is a chemical species that is essential for life and is involved in the most diverse range of chemical reactions and physical phenomena. Since oxygen present in the air allows the growth of molds and other strict aerobic microorganisms and oxidizes nutrients, such as fats and oils, it is one of the primary factors controlling food deterioration. ${ }^{1}$ Enormous efforts have been made to develop oxygen sensors and oxygen indicators that can easily detect oxygen gas. ${ }^{2-5}$ In contrast to oxygen sensors that are capable of detecting oxygen molecules and converting the detecting information into electrical signals in a quantitative manner, oxygen indicators are defined as devices that enable people to recognize changes in the optical absorbance of organic dyes or pigments according to the oxygen concentration in the form of changes in color in a semi-quantitative or a rather qualitative manner. Thus, from the viewpoint of detection, oxygen indicators can offer several advantages over oxygen sensors due to their immunity from electrical and electromagnetic interferences, durability, small size and their low cost.

Conventional oxygen indicators are known to use methylene blue (methyl thionine chloride (MB)), a dye that reversibly changes its color upon oxidation and reduction, as shown in Fig. 1. 6 Various oxygen indicators, such as ones in the form of a solid for the greater ease of handling, ${ }^{8}$ oxygen-detective ink composites $^{9}$ and their printed materials ${ }^{10}$ have been proposed. Actual commercially available tablet-type oxygen indicators

$\uparrow$ To whom correspondence should be addressed.

E-mail: inoue-haruo@c.metro-u.ac.jp and oxygen indicators in the form of strips of paper coated by an ink composites having an oxygen-detecting function are fabricated to contain an additional red dye or pigment, such as phloxine $\mathrm{B}$, in order to visually emphasize the color change. It enables them to exhibit a blue color in the presence of air and a red color in the absence of oxygen. These oxygen indicators are<smiles>CN(C)c1ccc2nc3ccc(=[N+](C)C)cc-3sc2c1</smiles>

Methylene Blue (MB), blue<smiles>CN(C)c1ccc2c(c1)Sc1cc(N(C)C)ccc1N2</smiles>

Leucomethylene Blue (LMB), colorless

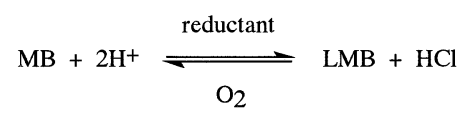

Fig. 1 Reduction and oxygen oxidation cycle of methylene blue. Blue-colored methylene blue (MB) is reduced by a reductant to form colorless leucomethylene blue (LMB), and then reverts to $\mathrm{MB}$ with its blue color due to oxidation by the oxygen molecule. 


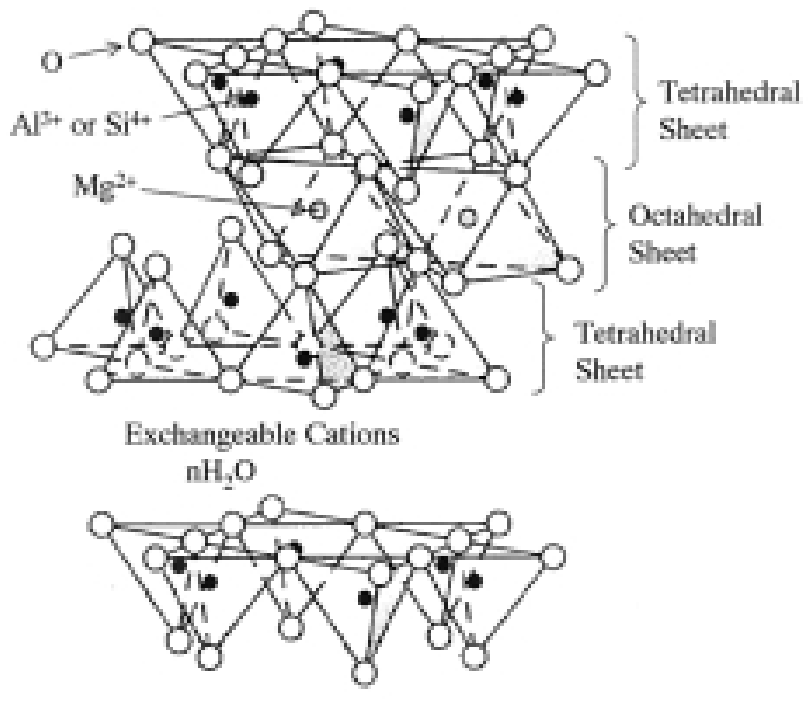

Fig. 2 Schematic structure of saponite.

functional products exhibiting a color change to indicate whether or not the inside of a transparent packaging container is an oxygen-free environment, and are used along with oxygen scavengers to maintain food freshness and to maintain the quality of pharmaceuticals and so forth. However, due to an insufficient resistant character against photo-fading and storage stability of current commercially available oxygen indicators, they become discolored, or suffer a decrease in their colorchanging function when stored while exposed to light and at room temperature, and are obliged to be stored in the dark and at low temperature in order to maintain their clear color-changes.

Nano-layered compounds, such as clay minerals, are well known to accommodate various organic molecules within their interlayer. The incorporation of molecules within the interlayer is termed "intercalation". The intercalated complex is called an "organic/inorganic hybrid compound". The intercalated molecules experience a different microenvironment from that in an ordinary solution, and are rather isolated from a bulk-phase solvent when the hybrid compound is dispersed in a solvent. They are, thus, expected to exhibit different characteristics against light irradiation ${ }^{11-15}$ and high temperature. ${ }^{16-23}$ Saponite is a layered clay mineral that has a large cation-exchange capacity, and easily forms organic/inorganic hybrid compounds with cationic organic substances. A schematic structure of the saponite is shown in Fig. 2. Layered clay minerals are also known to form stable compounds with cationic surfactants. ${ }^{11-15,24-27}$ These types of organic/inorganic hybrid compounds are thus expected to be potential candidates that can provide novel microenvironments for the oxygen indicator. From these viewpoints, in this paper we report on the fabrication of a new oxygen indicator using an organic/inorganic hybrid compound consisting of intercalated methylene blue (ionic form, $\mathrm{MB}^{+}$), a reductant and a cationic surfactant into the layers of saponite. The highly improved resistant character against light irradiation and storage stability of the oxygen indicator is also reported.

\section{Experimental}

Materials

"Sumecton SA" (Kunimine Industries), a synthetic clay saponite, was used as received. The chemical formula of
Table 1 Composition of oxygen-detective ink Liquid A (wt\%)

\begin{tabular}{lcrrr}
\hline \multicolumn{1}{c}{ Composition } & \multicolumn{1}{c}{1} & 2 & 3 & \multicolumn{1}{c}{4} \\
\hline MB & 0.10 & & & \\
MB(0.5)/CTAC(20.3)/ & & 20.00 & & \\
$\quad$ saponite(31.7)/MeOH(47.5) & & & & \\
MB(1.0)/CTAC(20.2)/ & & & 10.00 & 10.00 \\
$\quad$ saponite(31.5)/MeOH(47.3) & & & & \\
Phloxine B & 0.05 & 0.05 & 0.05 & 0.05 \\
Ethylene glycol & 22.30 & 22.30 & 22.30 & 22.30 \\
Cellulose acetate propionate & 6.24 & 6.14 & 6.24 & 5.97 \\
Ethyl acetate & 28.08 & 24.29 & 20.93 & 18.04 \\
Isopropyl alcohol & 28.08 & 12.29 & 20.48 & 18.04 \\
Fructose & 15.15 & 14.93 & 20.00 & 25.60 \\
\hline
\end{tabular}

Sumecton $\quad \mathrm{SA} \quad$ is $\quad\left[\left(\mathrm{Si}_{7.20} \mathrm{Al}_{0.80}\right)\left(\mathrm{Mg}_{5.97} \mathrm{Al}_{0.03}\right) \mathrm{O}_{20}(\mathrm{OH})_{4}\right]^{-0.77}$ $\left(\mathrm{Na}_{0.49} \mathrm{Mg}_{0.14}\right)^{+0.77}$. Its specific surface area is $750 \mathrm{~m}^{2} \mathrm{~g}^{-1}$, and the theoretical cationic-exchange capacity is $0.997 \mathrm{meq}^{-1}{ }^{28,29}$ Ultra-pure water with an electrical conductivity of $0.056 \mu \mathrm{S}$ $\mathrm{cm}^{-1}$, obtained with an ultra-pure water treatment system (RO60 (reverse osmosis membrane) + Milli-Q (activated charcoal + ion exchange + precision filtration) system) (Nihon Millipore), was used. "Yupo" polypropylene-based synthetic paper having a thickness of $70 \mu \mathrm{m}$, manufactured by Oji Paper, was used as synthetic paper on which the oxygen-detective ink was printed.

\section{Formation of organic/inorganic hybrid compounds}

An aqueous solution of $0.1 \mathrm{~N}$ sodium hydroxide was dropped into $20 \mathrm{~mL}$ of an aqueous mixture of $\mathrm{MB}^{+}\left(0.01 \mathrm{mmol} \mathrm{L}^{-1}\right)$, ascorbic acid or glucose $\left(2.0 \mathrm{mmol} \mathrm{L}^{-1}\right)$, cetyltrimethylammonium chloride (CTAC) $\left(2.0 \mathrm{mmol} \mathrm{L}^{-1}\right)$ and Sumecton SA $\left(0.2 \mathrm{meq} \mathrm{L}^{-1}\right)$ to adjust the $\mathrm{pH}$ of the solution to be 11.0 , followed by suction filtration (diameter, $35 \mathrm{~mm}$; pore size, $0.2 \mu \mathrm{m}$ ) of that solution to obtain an organic/inorganic hybrid compound in the form of a clear, blue thin film.

\section{Preparation of oxygen-detective ink pigments}

$\mathrm{MB}^{+}(0.16 \mathrm{~g}$ or $0.32 \mathrm{~g})$, CTAC $(6.4 \mathrm{~g})$, Sumeton SA (10.0 g) and methanol $(15 \mathrm{~mL})$ were mixed using a hybrid mixer (Keyence HM-500) to obtain an oxygen-detective ink pigment.

\section{Composition of oxygen-detective ink}

The ink composition of Liquid A (wt\%) is summarized in Table 1. In contrast to "Composition 1", containing only $\mathrm{MB}^{+}$ as a functional dye, "Compositions 2, 3, 4" contain $\mathrm{MB}^{+}$in the form of an organic/inorganic hybrid compound. In all cases of Compositions 1 through 4, the concentrations of $\mathrm{MB}^{+}$and phloxine $B$ were made to be the same, respectively. In Compositions 1 and 2, the concentrations of the fructose as the reductant were nearly equal to be $15 \mathrm{wt} \%$, while in Compositions 3 and 4 , the fructose concentrations were increased to $20 \mathrm{wt} \%$ and $26 \mathrm{wt} \%$, respectively. Liquid B contains magnesium hydroxide (43 wt $\%$ ), cellulose acetate propionate $(5 \mathrm{wt} \%)$, ethyl acetate (26 wt $\%)$, isopropyl alcohol (26 wt $\%)$. The composition of the filler was as follows: cyclized rubber resin (10 wt $\%)$, magnesium hydroxide (30 $\mathrm{wt} \%)$, toluene $(30 \mathrm{wt} \%)$, and cyclohexane $(30 \mathrm{wt} \%)$. An overprinting agent contained cyclized rubber resin (19 wt $\%$ ), sodium carbonate (3 wt \%), toluene (39 wt \%), and cyclohexane (39 wt \%).

\section{Analysis}

The interlayer distance of the organic/inorganic hybrid compounds was determined by X-ray diffraction measurements 
(Rigaku RINT2100). The amount of $\mathrm{MB}^{+}$intercalated into the hybrid compound was determined by the decrease in the absorbance of the $\mathrm{MB}^{+}$mother solution. The composition of the organic/inorganic hybrid compounds was determined by thermogravimetric analysis (Rigaku TAS300/TG8101) and elemental analysis for the total nitrogen (Mitsubishi Chemical TN-10). The absorption spectrum of the oxygen indicators was measured on an ultraviolet-visible spectrophotometer (Shimadzu UV-2500PC) equipped with an integrating sphere unit (Shimadzu ISR-2200).

\section{Evaluation of color changing performance}

(1) Anaerobic color changing performance was evaluated by a visual color change of the sample when it was stored at $25^{\circ} \mathrm{C}$ and $80 \% \mathrm{RH}$ in a sealed plastic container having a high barrier for gas penetration, together with a commercially available oxygen scavenger (Ageless SA (Mitsubishi Gas Chemical)). A zirconia oxygen concentration meter (Toray LC-700F) was used to monitor the oxygen concentration within the container. The amount of oxygen scavenger and the volume of the container were adjusted so that the oxygen concentration in the container reached $0.1 \mathrm{vol} \%$ after about $16 \mathrm{~h}$. (2) The aerobic colorchanging performance of the sample was evaluated by opening the plastic container, in which the oxygen concentration was less than $0.1 \mathrm{vol} \%$, to expose it to the air atmosphere. The color change of the sample was visually assessed.

\section{Results and Discussion}

\section{Organic/inorganic hybrid compound for detecting oxygen}

An organic/inorganic hybrid compound for detecting oxygen was prepared by intercalating $\mathrm{MB}^{+}$, a reductant and surfactant, cetyltrimethylammonium ion $\left(\mathrm{CTA}^{+}\right)$, into the layers of saponite. Ascorbic acid or basic glucose was used as the reductant. $\mathrm{MB}^{+}$can easily be intercalated into the layers of saponite by mixing it with an aqueous dispersion of saponite, since it is a cation, itself. The mixture of the $\mathrm{MB}^{+}$-intercalated hybrid compound with the reductant, however, did not show any color-change under nitrogen or upon exposure to air. This strongly suggests that the reductants can not react with $\mathrm{MB}^{+}$. They might not migrate into the interlayer space where $\mathrm{MB}^{+}$ exists. However ascorbic acid and glucose under basic conditions are anionic species. It would be reasonable that these anionic reductants can not penetrate into the interlayer space of the cation-exchangeable clay, saponite, due to the repulsive interaction between the anionic sites of the clay surface and the anionic reductant molecules. To more elegantly incorporate the anionic reductant species within the interlayer of the clay, saponite, on which surface anionic sites are delocalized, we further examined the co-intercalation of a cationic surfactant in the presence of $\mathrm{MB}^{+}$and the anionic reductant species. We have already found that anionic dye molecules can be successfully intercalated within the nanolayered space of niobate ${ }^{30}$ and saponite by the co-intercalation of cationic surfactants. The cationic surfactant would form an ion association complex with the anionic species, and the resulting complex is incorporated within the interlayer space where the cationic surfactants form a bilayer structure. The hydrophobic interaction between the long alkyl-chains of ionic association compound and the intercalated surfactants may prevail the electrostatically repulsive interaction between the anionic sites on the clay surface and the anionic species. From these viewpoints of strategy, organic/inorganic hybrid compounds were, thus, prepared by co-intercalation with the

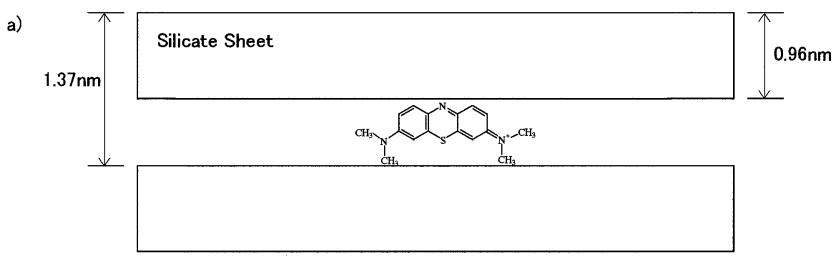

b)

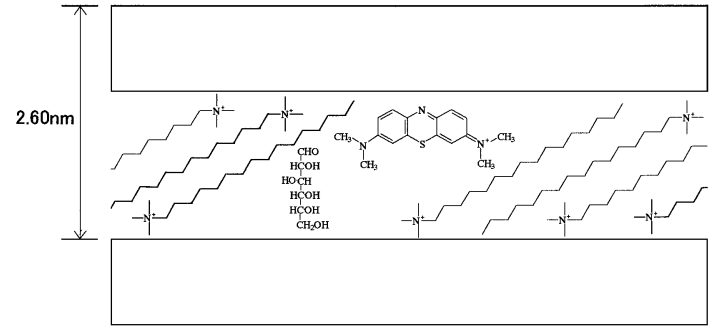

Fig. 3 Formation of an organic/inorganic hybrid compound having an oxygen-detection ability. a) Intercalation of methylene blue $\left(\mathrm{MB}^{+}\right)$being intercalated into saponite and b) co-intercalation of $\mathrm{MB}^{+}$ and reducing sugar with cetyltrimethylammonium ion $\left(\mathrm{CTA}^{+}\right)$.

cationic surfactant, CTAC, as shown in Fig. 3. The interlayer distance of the organic/inorganic hybrid compound (including the thicknesses of the layers) slightly increased from $1.31 \mathrm{~nm}$ to $1.37 \mathrm{~nm}$ when the aqueous dispersion of the clay was mixed with an aqueous solution of $\mathrm{MB}^{+}$, and further increased to 2.60 $\mathrm{nm}$ by co-intercalation as the result of intercalating $\mathrm{CTA}^{+}$. The organic/inorganic hybrid compound was found to consist of $0.056 \mathrm{~mol} \mathrm{MB}^{+}, 4.3 \mathrm{~mol}$ glucose and $1.2 \mathrm{~mol} \mathrm{CTA}^{+}$relative to $1.0 \mathrm{eq}$ of saponite. This organic/inorganic hybrid compound becomes nearly colorless and transparent in a deoxygenated atmosphere having an oxygen concentration of less than 0.1 vol\%, and rapidly reverts to its clear, blue color when it is exposed to air, as shown in Fig. 4. These distinct color changes of the hybrid compound was repeatedly observed under repeated exposure to deoxygenated and oxygenated atmospheres, indicating that the blue-colored $\mathrm{MB}^{+}$is reduced into the colorless LMB by the reductant under the deoxygenated atmosphere and is again reverted to $\mathrm{MB}^{+}$by oxygen under air, as expressed in the chemical equation in Fig. 1. A sufficient concentration of glucose as the reductant within the interlayer of the hybrid compound obtained by the co-intercalation method, as described above, renders the system possible to work well as an oxygen indicator.

\section{Oxygen indicator printed with oxygen-detective ink}

Composition and preparation of oxygen-detective ink. To fabricate an actual oxygen indicator, an oxygen-detective ink was prepared by blending the resin solution (see Experimental) with an organic/inorganic hybrid compound consisting of $\mathrm{MB}$, CTAC, saponite and fructose; the mixture was then coated onto a strip of paper. A red pigment, phloxine $\mathrm{B}$, was added to the above mixture in order to emphasize a visual color change upon exposure to the deaerated and aerated atmosphere. The oxygendetective ink was of the two-liquid type. After preparing a liquid in which an organic/inorganic hybrid compound composed of MB, CTAC and saponite, phloxine B and fructose were dispersed in a resin solution (Liquid A (Table 1)), and a liquid in which magnesium hydroxide was dispersed in a resin solution (Liquid B), the two liquids were mixed immediately 


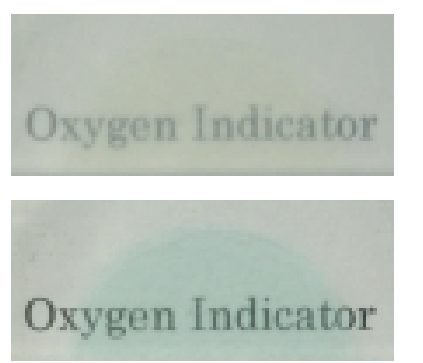

oxygen-free

under ambient air

for $1 \mathrm{~min}$

for $2 \mathrm{~min}$
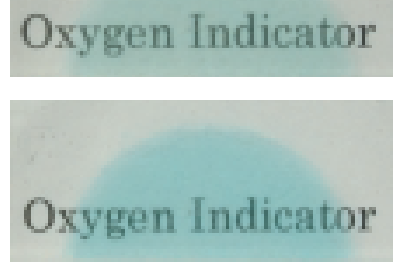

for $5 \mathrm{~min}$
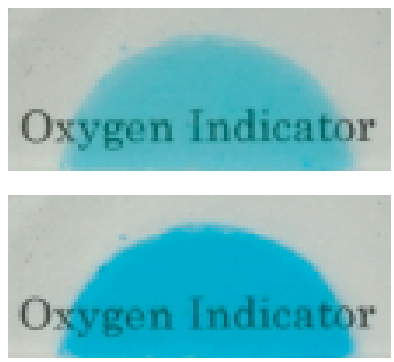

for $30 \mathrm{~min}$

for $60 \mathrm{~min}$

Fig. 4 Color change of an organic/inorganic hybrid compound upon exposure to air. The organic/inorganic hybrid compound was composed of $\mathrm{MB}^{+}(0.056 \mathrm{~mol})$, ascorbic acid $(4.3 \mathrm{~mol}), \mathrm{CTA}^{+}(1.2$ mol) and saponite $(1.0 \mathrm{eq})$. A semicircular film of the hybrid compound placed on a glass plate became colorless and transparent when subjected to an oxygen-free atmosphere having an oxygen concentration of less than $0.1 \mathrm{vol} \%$, and became blue when exposed to air. Photographs were taken by placing the film of the hybrid compound on a printed letters ("Oxygen Indicator").

just before printing. Polypropylene-based synthetic paper was used as the printed material, which was coated with a filler as the bottom layer, the oxygen-detective ink as the middle one, and an overprinting agent as the top one. The resulting printed material was dried at ambient temperature and cut into short pieces to obtain an oxygen indicator in the form of blue paper strips.

Color-changing performance of the oxygen indicator. The anaerobic and aerobic color-changing performance of the oxygen indicator paper strips fabricated by using different compositions of oxygen-detective ink were evaluated. The results for the different conditions are shown in Fig. 5. When the oxygen concentration inside the containers decreased from 20.9 vol\% to 0.1 vol\% within $16 \mathrm{~h}$, the oxygen indicators with Compositions 1 and 4 changed their colors from blue to purple, and then turned into pink color after $24 \mathrm{~h}$. On the other hand, the paper strip having Composition 2 was still bluish-purple even after $24 \mathrm{~h}$ and one with Composition 3 was purple. A much longer time was required for the paper strips having Compositions 2 and 3 to become pink. All the oxygen indicators that had once become pink returned to their original blue color within $5 \mathrm{~min}$ after exposure to air. When this procedure was repeated, the color of these oxygen indicators changed reversibly in response to the oxygen concentration.

\begin{tabular}{lcccc}
\hline Time (h) & 0 & 16 & 24 & 48 \\
Oxygen (vol\%) & 20.9 & 0.1 & $<0.1$ & $<0.1$ \\
\hline Composition 1 & & & & \\
Composition 2 & & & & \\
Composition 3 & & & & \\
Composition 4 & & & \\
\hline
\end{tabular}

Fig. 5 Color changes of oxygen indicators according to the oxygen concentration. Oxygen indicators fabricated by coating oxygendetective inks having different compositions (see Table 1) onto synthetic paper were sealed in transparent plastic containers along with an oxygen scavenger, and the color changes of the oxygen indicators were visually observed with the passage of time.

The sensitivity of these oxygen indicators was carefully examined by controlling the oxygen concentration, and it turned out that they exhibited pink color at an oxygen concentration of less than $0.1 \mathrm{vol} \%$, and reversibly exhibited a blue color at an oxygen concentration higher than $0.5 \mathrm{vol} \%$. The colorchanging performance of the indicator was not affected by the relative humidity.

Oxygen indicators fabricated by oxygen-detective ink containing $\mathrm{MB}^{+}$intercalated within the organic/inorganic hybrid compound (Compositions 2 and 3) exhibited a slower response for the color change under an anaerobic condition, as compared with the case of containing only $\mathrm{MB}^{+}$(Composition 1 ). The slower response is thought to be due to a limited diffusion of the reducing agent against $\mathrm{MB}^{+}$within the interlayer space of the hybrid compound. The response, however, was greatly improved to be very fast when the amount of fructose, as the reductant, was increased (Composition 4), which exhibited the best performance as the oxygen indicator among the examined cases, and would promisingly will serve as a commercial oxygen indicator.

\section{Stability and durability of the oxygen indicator}

An accelerated photodegradation test and storage-stability test were carried out on the oxygen indicators, as shown in Fig. 6. Paper-strip indicators, fabricated by using oxygen-detective ink with different compositions, were irradiated with visible light of $5000 \mathrm{~lx}$ using a fluorescent lamp as the light source in air at $25^{\circ} \mathrm{C}$ and $60 \% \mathrm{RH}$. The photo-fading of $\mathrm{MB}^{+}$was examined by observing any changes in the visible absorption spectrum (diffuse reflectance method). Here, the spectral intensities were expressed by normalizing the intensity at the maximum absorption wavelength of phloxine B. Composition 1 exhibited a relatively large degradation of $\mathrm{MB}^{+}$upon light irradiation, and became purple after $5 \mathrm{~h}$ of irradiation. Furthermore, the degradation of $\mathrm{MB}^{+}$during storage for a long time was also significant in the case of Composition 1. It turned into purple after 8 weeks of storage. On the other hand, Compositions 2 through 4 exhibited relatively very little degradation of $\mathrm{MB}^{+}$ upon light irradiation or storage for a long time, and maintained their blue color.

Oxygen indicators using $\mathrm{MB}$ incorporated within the organic/inorganic hybrid compound were shown to have superior light resistance and storage stability to the case of using MB alone as a functional pigment. The isolated microenvironment of the interlayer space of the clay, saponite, is considered to effectively retard the degradation of $\mathrm{MB}^{+}$, 

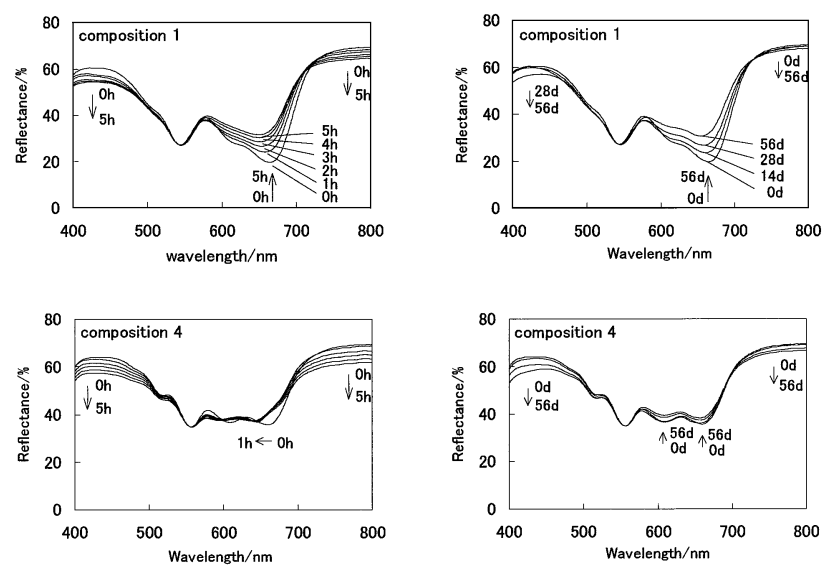

Fig. 6 Changes in the visible absorption spectra of oxygen indicators according to light irradiation time (left) and storage period (right). The visible absorption spectra of oxygen indicators fabricated by coating with oxygen-detective inks having different compositions (see Table 1) onto synthetic paper were observed. The spectral intensities were normalized at the maximum absorption wavelength of phloxine B (543 nm (Composition 1), $555 \mathrm{~nm}$ (Composition 4)). Light-irradiation conditions: fluorescent light at $5000 \mathrm{~lx}$, air, $25^{\circ} \mathrm{C}, 60 \% \mathrm{RH}, 5 \mathrm{~h}$. Storage conditions: dark location, air, $25^{\circ} \mathrm{C}, 60 \% \mathrm{RH}, 8$ weeks.

mainly by (1) a limited diffusion of oxygen molecules that can oxidatively induce the degradation of $\mathrm{MB}^{+}$through a formation of singlet oxygen generated by quenching of the excited $\mathrm{MB}^{+}$ within the interlayer; (2) a strong binding of the cationic $\mathrm{MB}^{+}$ on the negatively charged clay surface to prevent the D-D type photo-fading by suppressing the mutual collision of $\mathrm{MB}^{+}$within the interlayer; and (3) a limited diffusion of the third molecule, which might attack $\mathrm{MB}^{+}$either in the excited or ground state to induce degradation.

\section{Acknowledgements}

This work has been partly supported by a Grant-in-Aid for Exploratory Research and Scientific Research on Priority Areas (417) from the Ministry of Education, Culture, Sports, Science and Technology (MEXT) of the Japanese Government.

\section{References}

1. A. L. Brody, E. R. Strupinsky, and L. R. Kline, "Active Packaging for Food Applications", 2001, Technomic, Lancaster, 12.

2. Y. Amao, K. Asai, T. Miyashita, and I. Okura, Polymer J., 1999, 31(12), 1267.
3. Y. Amao, K. Asai, and I. Okura, Porphyrins, 1999, 8(2), 77.

4. C. Malins, S. Fanni, H. G. Glever, J. G. Vos, and B. D. MacCraith, Anal. Commun., 1999, 36, 3.

5. M. T. Murtagh, M. R. Shahriari, and M. Krihak, Chem Mater., 1998, 10, 3862.

6. M. V. Riemsdijk, Nederland. Tijdschr. Geneeskunde, 1922, 66, I, 1423.

7. W. M. Clark and B. Cohen, Abstracts Bact., 1925, 9, 11.

8. Y. Yoshikawa, Y. Fujii, T. Nawata, and M. Goto, a) Japan Patent, 1981, 1052402; b) Japan Patent, 1984, 1218096; c) U. S. Patent, 1979, 4169811.

9. M. Nakamoto and K. Omoto, Japan Patent, 1988, 1449258.

10. Y. Yoshikawa, T. Nawata, M. Goto, and Y. Kondo, U. S. Patent, 1982, 4349509.

11. J. K. Thomas, Acc. Chem. Res., 1988, 21, 275.

12. J. K. Thomas, Chem. Rev., 1993, 93, 301.

13. M. Ogawa and K. Kuroda, Chem. Rev., 1995, 95, 399.

14. K. Takagi and T. Shichi, in "Solid State and Surface Photochemistry", ed. V. Ramamurthy and K. S. Schanze, 2000, Vol. 5, Marcel Dekker, New York, 31.

15. K. Takagi and T. Shichi, J. Photochem. Photobiol. C: Photochem. Rev., 2000, 1, 112.

16. C. Kato, a) Koubunshi, 1979, 28, 575; b) Japan Patent, 1975, 0795548; c) U. S. Patent, 1976, 3950180.

17. T. Itoh, K. Yano, Y. Inada, and Y. Fukushima, J. Mater. Chem., 2002, 12, 3275.

18. T. Itoh, K. Yano, Y. Inada, and Y. Fukushima, J. Am. Chem. Soc., 2002, 124, 13437.

19. H. Rozen and L. Margulies, J. Agric. Food Chem., 1991, 39, 1320.

20. M. A. Caine, R. W. McCabe, L. Wang, R. G. Brown, and J. D. Hepworth, Dyes Pigm., 2002, 52, 55.

21. K. Banerjee and P. Dureja, Pestic. Sci., 1995, 43, 333.

22. L. Margulies, H. Rozen, T. Stern, G. Rytwo, B. Rubin, L. O. Ruzo, S. Nir, and E. Cohen, Arch. Insect Biochem. Physiol., 1993, 22, 467.

23. L. Margulies, H. Rozen, and E. Cohen, Clays Clay Miner. 1988, 36, 159.

24. L. A. Lucia, T. Yui, R. Sasai, S. Takagi, K. Takagi, H. Yoshida, D. G. Whitten, and H. Inoue, J. Phys. Chem. B, 2003, 107, 3789.

25. T. Yui, S. R. Uppili, T. Shimada, D. A. Tryk, H. Yoshida, and H. Inoue, Langmuir, 2002, 18, 4232.

26. S. Xu and S. A. Boyd, Langmuir, 1995, 11, 2508.

27. Y. Sakai, N. Kato, M. Arai, F. Takahashi, and Y. Nishida, Bull. Chem. Soc. Jpn., 2002, 75, 2403.

28. S. Takagi, T. Shimada, T. Yui, and H. Inoue, Chem. Lett., 2001, 128.

29. S. Takagi, T. Shimada, M. Eguchi, T. Yui, H. Yoshida, D. A. Tryk, and H. Inoue, Langmuir, 2002, 18, 2265.

30. Y. Yamaguchi, Y. Yui, S. Takagi, T. Shimada, and H. Inoue, Chem. Lett., 2001, 644. 